\title{
Erratum to: Do We Need another Antiangiogenesis Agent for Colorectal Cancer: are Bevacizumab and Aflibercept the Same?
}

\author{
Maria Ignez Braghiroli • Rachel P. Riechelmann • \\ Jorge Sabbaga $\cdot$ Paulo M. Hoff
}

Published online: 28 May 2014

(C) Springer Science+Business Media New York 2014

Erratum to: Curr Colorectal Cancer Rep (2013) 9:317-325

DOI 10.1007/s11888-013-0187-0

The original version of this article, published in Current Colorectal Cancer Reports, Volume 9, Issue 4, December 2013, contained a misprint in Table 1, "Comparison of bevacizumab and aflibercept." Under "Dose and association in colorectal cancer," the dosing unit for bevacizumab was given in " $\mathrm{mg} / \mathrm{m}^{-2}$," when it should be " $\mathrm{mg} / \mathrm{kg}$." The table provided is now correct.

The online version of the original article can be found at http://dx.doi.org/ 10.1007/s11888-013-0187-0.

M. I. Braghiroli $(\bowtie) \cdot$ R. P. Riechelmann · J. Sabbaga $\cdot$ P. M. Hoff Centro de Oncologia do Hospital Sírio Libanês, Rua D. Adma Jafet, 91, Bela Vista CEP, 01308-050 São Paulo, SP, Brazil

e-mail: ignezbraghiroli@gmail.com

R. P. Riechelmann

e-mail: rachel.riechelmann@icesp.org.br

J. Sabbaga

e-mail: jsabbaga@uol.com.br

P. M. Hoff

e-mail: paulo.hoff@icesp.org.br

M. I. Braghiroli • J. Sabbaga $・$ P. M. Hoff Instituto de Câncer do Estado de São Paulo do Hospital das Clínicas da Faculdade de Medicina de Universidade de São Paulo, Av. Doutor Amaldo, 251, Oncologia Clínica $5^{\circ}$ andar, Cerqueira César CEP, 01246-000 São Paulo, SP, Brazil 
Table 1 Comparison of bevacizumab and aflibercept

\begin{tabular}{|c|c|c|}
\hline & Bevacizumab & Aflibercept \\
\hline Description & $\begin{array}{l}\text { Humanized monoclonal immunoglobulin } \mathrm{G} \\
\text { antibody }\end{array}$ & $\begin{array}{l}\text { Fully humanized soluble recombinant fusion protein with domains } \\
\text { from VEGFR-1 and VEGFR-2 }\end{array}$ \\
\hline Target & VEGF-A & VEGF-A, VEGF-B, PIGF \\
\hline Molecular weight & $149 \mathrm{kD}$ & $115 \mathrm{kD}$ \\
\hline $\begin{array}{l}\text { Dose and association in } \\
\text { colorectal cancer }\end{array}$ & $\begin{array}{l}\text { 5-10 mg/kg, IV, every } 2 \text { weeks with FOLFOX } \\
\text { or FOLFIRI }\end{array}$ & $4 \mathrm{mg} / \mathrm{kg}$ every 2 weeks with FOLFIRI \\
\hline Relevant adverse events & $\begin{array}{l}\text { Hemorrhage, thrombosis, proteinuria, } \\
\text { hypertension, GI perforation }\end{array}$ & Hemorrhage, hand-foot syndrome, hypertension, fatigue \\
\hline $\begin{array}{l}\text { Worsening chemotherapy } \\
\text { side effects }\end{array}$ & No & Yes, specially diarrhea, neutropenia, stomatitis \\
\hline Positive Phase III Trials & $\begin{array}{l}\text { First Line: } \\
\text { IFL+/- bevacizumab } \\
\text { FOLFOX/XELOX +/- bevacizumab } \\
\text { Second Line: } \\
\text { FOLFOX +/- bevacizumab } \\
\text { Chemotherapy +/- bevacizumab after } \\
\text { progression on bevacizumab }\end{array}$ & $\begin{array}{l}\text { Second Line } \\
\text { FOLFIRI +/- aflibercept }\end{array}$ \\
\hline
\end{tabular}

